\title{
Cyclodextrin-Enclosed Substances of Brazilian Propolis
}

\author{
Alaa Mohamed Nafady, ${ }^{a}$ Mohamed Ahmed El-ShanawanY, ${ }^{b}$ Mahmoud Hamed Mohamed, ${ }^{c}$ \\ Hashim Abdel-Halim Hassanean, ${ }^{b}$ Toshihiro Nohara, ${ }^{* a}{ }^{, a}$ Hitoshi Yoshimitsu, ${ }^{d}$ Masateru Ono, ${ }^{a}$ \\ Hiroyuki Sugimoto, ${ }^{e}$ Shima Doi, ${ }^{e}$ Ken SAsaki, ${ }^{f}$ and Hirohisa KurodA ${ }^{f}$ \\ ${ }^{a}$ Faculty of Pharmaceutical Sciences, Kumamoto University; 5-1 Oe-honmachi, Kumamoto, 862-0973, Japan: \\ ${ }^{b}$ Department of Pharmacognosy, Faculty of Pharmacy, Assiut University; Assiut, Egypt: ${ }^{c}$ Department of Pharmacognosy, \\ Faculty of Pharmacy, Al-Azhar University, Assiut Branch; Assiut, Egypt: ${ }^{d}$ Faculty of Engineering, Kyushu Kyoritsu \\ University; 1-8 Jiyugaoka, Yahata-nishi-ku, Kitakyushu 807-8585, Japan: ${ }^{e}$ Yamada Apiculture Center, Inc.; 194 Ichiba, \\ Kagamino, Tomata, Okayama 708-0393, Japan: and ${ }^{f}$ Department of Polymer Science \& Engineering, Kyoto Institute of \\ Technology; Matsugasaki, Sakyo-ku, Kyoto 606-8585, Japan. $\quad$ Received January 23, 2003; accepted April 21, 2003
}

By using $\beta$-cyclodextrin-inclusion as a unique technique, an efficient separation of pharmacologically active phenolic compounds from Brazilian propolis was achieved to provide one new compound, 3-(3-hydroxy-3methyl-butyl)-5-prenyl-4-hydroxycinnamic acid, together with two common cinnamic acid derivatives, artepillin $C$ and capillartemisin $A$, and two known flavanols, aromadendrin and 3,5,7-trihydroxy-4' -methoxyflavanol.

Key words Brazilian propolis; $\beta$-cyclodextrin-inclusion; 4-hydroxycinnamic acid derivative

Propolis is a resinous hive product collected by honeybees from various plant sources. ${ }^{1)}$ It is extensively used in food and beverages and in folk medicine for the treatment of different ailments, and is reported to have a broad spectrum of pharmacological activities such as anti-microbial activity, antioxidant, anticancer and as an immune stimulant in addition to other pharmacological effects. ${ }^{2,3)}$

Concerning the chemical composition of propolis, it turned out to be very complex, and more than 200 compounds have been isolated so far. ${ }^{2)}$ The most important constituent appears to be phenolics, which form more than $c a$. $50 \%$ of the propolis composition. ${ }^{4)}$ Since isolation of the bioactive substances is accompanied by many difficulties owing to their complexity and scarce amounts, we devised a method to obtain the aromatic compounds by using $\beta$-cyclodextrininclusion. In the present paper we describe the isolation and structural elucidation of some phenolic compounds isolated from Brazilian propolis by using $\beta$-cyclodextrin-inclusion as a selective method for the isolation of the phenolic constituents from the propolis extract.

Brazilian propolis was suspended in dist. water containing $\beta$-cyclodextrin. After the suspension was subjected to sonication, the insoluble propolis was filtered off. The water-soluble filtrate was concentrated by evaporation under reduced pressure to provide $\beta$-cyclodextrin-propolis inclusion, to which ethanol was added and vigorously stirred. The insoluble ethanol portion containing $\beta$-cyclodextrin was filtered off to give the ethanol solution, which was then concentrated to give the propolis extract. The propolis extract was subjected to Diaion HP-20, Sephadex LH-20, ODS and HPLC (ODS and TSK gel-120A) to give compounds $\mathbf{1}-\mathbf{5}$. Compounds $\mathbf{1}$, $\mathbf{2 , 4}$, and $\mathbf{5}$ were identified with artepilin $C,{ }^{5)}$ capillartermisin $\mathrm{A},{ }^{6}$ aromadendrin ${ }^{7-9)}$ and 3,5,7-trihydroxy-4'-methoxyflavanol,${ }^{10)}$ respectively, by spectroscopic measurements.

Compound 3 was obtained as a white powder showing $[\alpha]_{\mathrm{D}}-14.8^{\circ}(\mathrm{MeOH})$. It showed a molecular peak at $\mathrm{m} / \mathrm{z} 318$ $\left[\mathrm{C}_{19} \mathrm{H}_{26} \mathrm{O}_{4}\right]^{+}$in the electron impact (EI-MS). In the ${ }^{1} \mathrm{H}-\mathrm{NMR}$ spectrum, a set of prenyl signals was observed at $\delta 5.58(1 \mathrm{H}$, t-like), $3.66(2 \mathrm{H}, \mathrm{d}, J=7.3 \mathrm{~Hz}), 1.69$ and 1.68 each $(3 \mathrm{H}, \mathrm{s})$. It also showed the presence of two olfenic protons at $\delta 6.91$ and 8.10 each coupled with a large coupling constant $(1 \mathrm{H}, \mathrm{d}$,

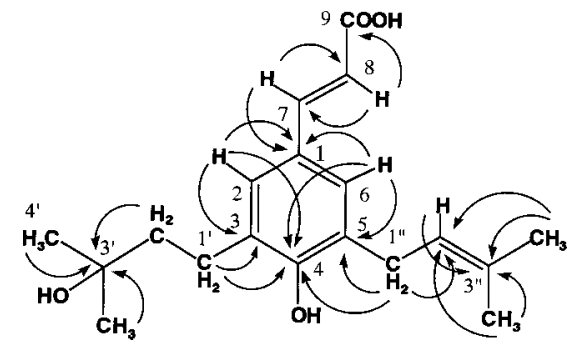

Fig. 1. Structure and Key HMBC of Compound 3

$J=15.9 \mathrm{~Hz}$ ), suggesting it to be a trans-double bond in a side chain. The ${ }^{13} \mathrm{C}$-NMR spectrum of $\mathbf{3}$ indicated the presence of 19 -carbon atoms composed of four methyls $(\delta 17.8,25.8$, $2 \times 29.8)$, three methylenes $(\delta 25.9,29.6,44.4)$, ten $s p^{2}$ carbons $(\delta 116.8,123.8,128.3,127.0,128.2,130.1,131.4$, $132.5,145.3,156.3)$ and one carbonyl carbon $(\delta 169.8)$. The two dimensional (2D)-NMR spectra of proton-proton chemical shift correlation spectroscopy $\left({ }^{1} \mathrm{H}-{ }^{1} \mathrm{H}\right.$ COSY $)$, heteromolecular multiple quantum coherence (HMQC) and heteronuclear multiple bond connectivity (HMBC) were observed for the assignment of ${ }^{1} \mathrm{H}$ and ${ }^{13} \mathrm{C}$ signals. The result of the HMBC spectrum is illustrated in Fig. 1. That is, the structure of $\mathbf{3}$ was a derivative of 3,5-disubstituted 4-hydroxycinnamic acid, and a tertiary hydroxyl group was attached to C- $3^{\prime}$ on the isopropyl moiety. Consequently, 3 was characterized as 3(3-hydroxy-3-methyl-butyl)-5-prenyl-4-hydroxycinnamic acid, which has not yet been reported.

\section{Experimental}

Optical rotations were determined on a JASCO DIP-1000 KUY polarimeter $(l=5 \mathrm{~cm})$. EI-MS was obtained using a JEOL JMS-DX300. NMR spectra were measured in pyridine- $d_{5}$ on a JEOL $\alpha-500$ spectrometer, and chemical shifts were referenced to tetramethylsilane (TMS). Column chromatography was carried out with silica gel 60 (230 - 400 mesh, Merck), Sephadex LH20 (25-100 mm, Pharmacia Fine Chemicals), MCI gel CHP-20P (75$150 \mathrm{~mm}$, Mitsubishikasei), Chromatorex ODS $(30-50 \mathrm{~mm}$, Fuji Silysia Chemical, Ltd.), and TLC was performed on precoated silica gel $60 \mathrm{~F}_{254}$ (0.2 mm, Merck) and RP-18 F254S (Merck). HPLC was performed on ODS gel (TSK gel-120A, Tosoh Co., Ltd. $\Phi=7.7 \mathrm{~mm}, L=300 \mathrm{~mm}$ ).

Isolation of Compounds $\mathbf{1}-\mathbf{5}$ from Brazilian Propolis Using $\boldsymbol{\beta}$-Cyclodextrin-Inclusion Brazilian propolis $(10 \mathrm{~g})$, which is regarded to be 
collected mainly from Baccharis dracunculifolia, Compositae, in the Minas Gerais region in Brazil, was suspended in 11 of dist. water, which contained $10 \mathrm{~g}$ of $\beta$-cyclodextrin. After the suspension was subjected to sonication for $4 \mathrm{~h}$, the insoluble propolis was filtered off. The water-soluble filtrate was concentrated by evaporation under reduced pressure to provide $\beta$-cyclodextrin-propolis inclusion, to which $700 \mathrm{ml}$ of ethanol was added and vigorously stirred. The insoluble ethanol portion containing $\beta$-cyclodextrin was filtered off to give the ethanol solution, which was then concentrated to give the propolis extract. The propolis extract $(1.054 \mathrm{~g})$ was subjected to Diaion HP20 , and successively flowed out with 20, 30, 50, 80 and $100 \% \mathrm{MeOH}-\mathrm{H}_{2} \mathrm{O}$ to give 4 fractions. Fraction $2(0.467 \mathrm{~g})$ was subjected to Sephadex LH-20 column chromatography using methanol to give two main fractions. Fraction 2.1 was chromatographed through ODS column chromatography using $\mathrm{MeOH}-\mathrm{H}_{2} \mathrm{O}(50,60,70 \%$, successively) to give 2 fractions. Fraction 2.1.2 was further chromatogarphed through HPLC (ODS) using the $\mathrm{MeOH}-\mathrm{H}_{2} \mathrm{O}$ system $(60 \%)$ to give Compound $1(21.3 \mathrm{mg})$. Fraction 2.2 was also chromatographed through ODS by $\mathrm{MeOH}-\mathrm{H}_{2} \mathrm{O}(50,60 \%)$ to give 2 fractions. Fraction 2.2.1 was further chromatographed by HPLC (ODS) using 50\% $\mathrm{MeOH}-\mathrm{H}_{2} \mathrm{O}$ to give compound $4(11.2 \mathrm{mg})$. Fraction $3(0.128 \mathrm{~g})$ was chromatographed through Sephadex $\mathrm{LH}-20$ using $\mathrm{MeOH}$ to give 2 main fractions. Fraction 3.1 was chromatographed by ODS column chromatography and $\mathrm{MeOH}-\mathrm{H}_{2} \mathrm{O}$ systems $(60,70,80 \%)$ gradiently to give 2 fractions. Fractions 3.1.1 and 3.1.2 were further chromatographed through HPLC [column TSK gel-120A] using a $70 \% \mathrm{MeOH}-\mathrm{H}_{2} \mathrm{O}$ system to give compounds 2 (13.4 mg) and 3 (10.4 mg), respectively. Fraction 3.2 was chromatographed through ODS column chromatography using the $\mathrm{MeOH}-\mathrm{H}_{2} \mathrm{O}$ system $(60$, $70 \%$ ) to give fraction 3.2 .1 , which was further chromatographed through HPLC (ODS) using a $60 \% \mathrm{MeOH}-\mathrm{H}_{2} \mathrm{O}$ system to give compound 5 (9.4 mg).

Compound $1{ }^{1} \mathrm{H}-\mathrm{NMR}$ (in pyridine- $\left.d_{5}\right) \delta: 1.71\left(6 \mathrm{H}, \mathrm{s}, \mathrm{H}_{3}-5^{\prime}, 5^{\prime \prime}\right), 1.74$ $\left(6 \mathrm{H}, \mathrm{s}, \mathrm{H}_{3}-4^{\prime}, 4^{\prime \prime}\right), 3.65$ (2H, d, J=7.3 Hz, H2-1', 1"), 5.62 (2H, t-like, H-2', 2"), $6.86(1 \mathrm{H}, \mathrm{d}, J=15.9 \mathrm{~Hz}, \mathrm{H}-8), 7.67(2 \mathrm{H}, \mathrm{s}, \mathrm{H}-2,6), 8.15(1 \mathrm{H}, \mathrm{d}$, $J=15.9 \mathrm{~Hz}, \mathrm{H}-7) .{ }^{13} \mathrm{C}-\mathrm{NMR}$ (in pyridine- $\left.d_{5}\right) \delta: 17.8\left(\mathrm{C}-5^{\prime}, 5^{\prime \prime}\right), 25.8\left(\mathrm{C}-4^{\prime}\right.$, 4"), 29.2 (C-1', 1"), 116.0 (C-8), 123.3 (C-2', 2"), 126.6 (C-1), 127.9 (C-2, 6), 129.9 (C-3, 5), 132.3 (C-3', 3"), 144.9 (C-7), 158.9 (C-4), 169.8 (C-9). Compound 1 was identified with artepillin $\mathrm{C}^{5)}$

Compound $2{ }^{1} \mathrm{H}-\mathrm{NMR}$ (in pyridine- $\left.d_{5}\right) \delta: 1.68\left(6 \mathrm{H}, \mathrm{s}, \mathrm{H}_{3}-4^{\prime \prime}, 5^{\prime \prime}\right), 1.90$ $\left(3 \mathrm{H}, \mathrm{s}, \mathrm{H}_{3}-5^{\prime}\right), 3.68\left(2 \mathrm{H}, \mathrm{d}, J=6.7 \mathrm{~Hz}, \mathrm{H}_{2}-1^{\prime \prime}\right), 3.79\left(2 \mathrm{H}, \mathrm{d}, J=7.3 \mathrm{~Hz}, \mathrm{H}_{2}-1^{\prime}\right)$, $4.32\left(2 \mathrm{H}, \mathrm{s}, \mathrm{H}_{2}-4^{\prime}\right), 5.56\left(1 \mathrm{H}, \mathrm{t}, \mathrm{H}-2^{\prime \prime}\right), 6.14\left(1 \mathrm{H}, \mathrm{t}, \mathrm{H}-2^{\prime}\right), 6.88(1 \mathrm{H}, \mathrm{d}$, $J=15.9 \mathrm{~Hz}, \mathrm{H}-8), 7.55(2 \mathrm{H}, \mathrm{d}, J=2.4 \mathrm{~Hz}, \mathrm{H}-2,6), 8.14(1 \mathrm{H}, \mathrm{d}, J=15.9 \mathrm{~Hz}$, $\mathrm{H}-7) .{ }^{13} \mathrm{C}-\mathrm{NMR}$ (in pyridine- $\left.d_{5}\right) \delta$ : $14.1\left(\mathrm{C}-3^{\prime}\right), 17.8\left(\mathrm{C}-5^{\prime \prime}\right), 25.7\left(\mathrm{C}-4^{\prime \prime}\right)$, 29.3, 29.6 (C-1', 1") $67.8\left(\mathrm{C}-4^{\prime}\right), 117.1$ (C-8), 123.1 (C-2'), 123.8 (C-2"), 127.1 (C-1), 128.1, 128.3 (C-2, 6), 129.9, 130.0 (C-3, 5), 145.2 (C-7). 169.8
(C-9). Compound 2 was identified with capillartemisin A. ${ }^{6}$

Compound 3 A white powder, $[\alpha]_{\mathrm{D}}-14.8^{\circ}(c=0.25, \mathrm{MeOH})$, EI-MS $(m / z): 318\left[\mathrm{M}, \mathrm{C}_{19} \mathrm{H}_{26} \mathrm{O}_{4}\right]^{+},{ }^{1} \mathrm{H}-\mathrm{NMR}$ (in pyridine- $\left.d_{5}\right) \delta: 1.42\left(6 \mathrm{H}, \mathrm{s}, \mathrm{H}_{3}-4^{\prime}\right.$, $\left.5^{\prime}\right), 1.68\left(3 \mathrm{H}, \mathrm{s}, \mathrm{H}_{3}-5^{\prime \prime}\right), 1.69\left(3 \mathrm{H}, \mathrm{s}, \mathrm{H}_{3}-4^{\prime \prime}\right), 2.06\left(2 \mathrm{H}, \mathrm{m}, \mathrm{H}_{2}-2^{\prime}\right), 3.19(2 \mathrm{H}$, $\left.\mathrm{m}, \mathrm{H}_{2}-1^{\prime}\right), 3.66\left(2 \mathrm{H}, \mathrm{d}, J=7.3 \mathrm{~Hz}, \mathrm{H}_{2}-1^{\prime \prime}\right), 5.58\left(1 \mathrm{H}, \mathrm{t}, \mathrm{H}-2^{\prime \prime}\right), 6.91(1 \mathrm{H}, \mathrm{d}$, $J=15.9 \mathrm{~Hz}, \mathrm{H}-8), 7.54(2 \mathrm{H}, \mathrm{s}, \mathrm{H}-2,6), 8.10(1 \mathrm{H}, \mathrm{d}, J=15.9 \mathrm{~Hz}, \mathrm{H}-7) .{ }^{13} \mathrm{C}-$ NMR (in pyridine- $\left.d_{5}\right) \delta: 17.8\left(\mathrm{C}-5^{\prime \prime}\right), 25.8\left(\mathrm{C}-1^{\prime}\right), 25.9\left(\mathrm{C}-4^{\prime \prime}\right), 29.6\left(\mathrm{C}-1^{\prime \prime}\right)$, 29. 8 (C-4', 5'), 44.4 (C-2'), 69.9 (C-3'), 116.8 (C-8), 123.8 (C-2"), 128.3 (C-2), 127.0 (C-1), 128.2 (C-6), 130.1 (C-5), 131.4 (C-3), 132.5 (C-3"), 145.3 (C-7), 156.3 (C-4), 169.8 (C-9). Anal. Calcf for $\mathrm{C}_{19} \mathrm{H}_{26} \mathrm{O}_{4}$ : C, 71.67; H, 8.23; Found: C, 71.69; H, 8.21.

Compound $4{ }^{1} \mathrm{H}-\mathrm{NMR}$ (in pyridine- $\left.d_{5}\right) \delta: 4.73(1 \mathrm{H}, \mathrm{d}, J=11.6 \mathrm{~Hz}, \mathrm{H}-$ 3), $5.28(1 \mathrm{H}, \mathrm{d}, J=11.6 \mathrm{~Hz}, \mathrm{H}-2), 5.87(1 \mathrm{H}, \mathrm{d}, J=2.4 \mathrm{~Hz}, \mathrm{H}-6), 5.90(1 \mathrm{H}, \mathrm{d}$, $J=2.4 \mathrm{~Hz}, \mathrm{H}-8), 7.25\left(2 \mathrm{H}, \mathrm{d}, J=8.5 \mathrm{~Hz}, \mathrm{H}-3^{\prime}, 5^{\prime}\right), 7.74(2 \mathrm{H}, \mathrm{d}, J=8.5 \mathrm{~Hz}, \mathrm{H}-$ $2^{\prime}, 6^{\prime}$ ). ${ }^{13} \mathrm{C}-\mathrm{NMR}$ (in pyridine- $d_{5}$ ) $\delta: 84.6,73.3,198.9,165.1,97.5,168.8$, $96.2,163.1,101.7,128.9,130.2,116.0,159.6,128.9,130.2$. Compound 4 was identified with aromadendrin. ${ }^{7-9)}$

Compound $5{ }^{1} \mathrm{H}-\mathrm{NMR}$ (in pyridine- $\left.d_{5}\right) \delta: 4.98(1 \mathrm{H}, \mathrm{d}, J=11.0 \mathrm{~Hz}, \mathrm{H}-$ 3), $5.45(1 \mathrm{H}, \mathrm{d}, J=11.0 \mathrm{~Hz}, \mathrm{H}-2), 6.38(1 \mathrm{H}, \mathrm{d}, J=1.8 \mathrm{~Hz}, \mathrm{H}-6), 6.52(1 \mathrm{H}, \mathrm{d}$, $J=1.8 \mathrm{~Hz}, \mathrm{H}-8), 7.06\left(2 \mathrm{H}, \mathrm{d}, J=7.9 \mathrm{~Hz}, \mathrm{H}-3^{\prime}, 5^{\prime}\right), 7.74(2 \mathrm{H}, \mathrm{d}, J=8.5 \mathrm{~Hz}, \mathrm{H}-$ $\left.2^{\prime}, 6^{\prime}\right) .{ }^{13} \mathrm{C}-\mathrm{NMR}$ (in pyridine- $d_{5}$ ) $\delta: 84.3,73.3,198.5,165.1,97.5,168.8$, $96.3,163.8,101.6,130.5,130.0,114.3,160.5,130.0,55.3$. Compound $\mathbf{5}$ was identified with $3,5,7$-trihydroxy-4' ${ }^{\prime}$ methoxyflavanol. ${ }^{10)}$

\section{References}

1) Banskota A. H., Tezuka Y., Prasain J. K., Saiki I., Kadota S., J. Nat. Prod., 61, 896-900 (1998).

2) Marcucci M. C., Apidologie, 26, 83-99 (1995)

3) Burdock G. A., Food and Chem. Toxicol., 36, 347-363 (1998).

4) Bankova V., Marcucci M. C., Simova S., Nikolova N., Kujumgiev A., Popov S., Z. Naturforsch., 51, 277-280 (1996).

5) Okuno I., Uchida K., Nakamura M. Sakurawi K., Chem. Pharm. Bull., 36, 769-775 (1988).

6) Kitagawa I., Fukuda Y., Yoshihara M., Yamahara J., Yoshikawa M., Chem. Pharm. Bull., 31, 352-355 (1983).

7) El-Sohly H. N., Joshi A., Li X.-C., Ross S. A., Phytochemistry, 52, $141-145$ (1999).

8) Binutu O. A., Cordell G. A., Phytochemistry, 56, 827-830 (2001).

9) Tazawa S., Warashina T., Noro T., Miyase T., Chem. Pharm. Bull., 46, 1477-1479 (1998).

10) Banskota A. H., Tezuka Y., Prasain J. K., Matsushige K., Saiki I., Kadota S., J. Nat. Prod., 61, 896-900 (1998). 